\title{
Cyclic plasticity and creep-cyclic plasticity behaviours of the SiC/Ti-6242 Particulate Reinforced Titanium Matrix Composites under thermo-mechanical loadings
}

\author{
Dario Giugliano ${ }^{1,+}$, Nak-Kyun Cho ${ }^{1,+}$, Haofeng Chen ${ }^{1,2, *}$, Lorenzo Gentile ${ }^{3}$ \\ ${ }^{1}$ Department of Mechanical \& Aerospace Engineering, University of Strathclyde, Glasgow, G1 1XJ, UK \\ ${ }^{2}$ School of Mechanical and Power Engineering, East China University of Science and Technology, 200237, \\ China \\ ${ }^{3}$ Institute for Data Science, Engineering and Analytics, TH Köln, Gummersbach, 51643, DE \\ *Email: haofeng.chen@strath.ac.uk \\ ${ }^{+}$Both authors contributed equally to this work
}

\begin{abstract}
The purpose of this work is to investigate the cyclic plasticity and creep-cyclic plasticity behaviours of particle reinforced titanium matrix composites (PRTMCs) SiC/Ti-6242, aimed to be used in high temperature applications. The investigation has been conducted upon microstructures that have been taken from a previous study where low-fidelity model-based optimization (LFMBO) has been used to maximise the elastic behaviour of particle reinforced aluminium matrix composites. The effect of the particle spatial distribution, particle fraction volume and number of particles on the shakedown limit, limit load and creep-cyclic plasticity have been explored by direct numerical techniques based on the Linear Matching Method (LMM) framework. The micromechanical approach to modelling and fifteen multi-particle unit cells have been investigated. Under cyclic loading conditions, the structural response of PRTMCs is not trivial and becomes even more significant when high temperature is involved. Hence, the factors that affect the creep and cyclic plasticity of PRTMCs are analysed and discussed, including effects of the applied load level, dwell period and temperature on the composites' performance. The applicability and accuracy of the proposed direct method has also been verified by the step-by-step analysis.
\end{abstract}

Keywords: Shakedown, Creep-cyclic plasticity interaction, Particulate Reinforced Titanium Matrix Composites (PRTMCs), Reverse plasticity, Creep ratchetting 


\section{Introduction}

In the search of developing materials which are stronger, stiffer, lighter and capable of use at high temperature, over the past 50 years researchers have conducted an investigation on an extensive range of reinforcement/matrix combinations in metal matrix composites (MMCs). For both family of MMCs, i.e. continuous fibre reinforced MMCs (CFMMCs) and discontinuous MMCs (DMMCs) a wide spectrum of matrix materials (including aluminium $(\mathrm{Al})$, magnesium $(\mathrm{Mg})$, copper $(\mathrm{Cu})$, titanium $(\mathrm{Ti})$ and steel (Fe) among others), and ceramic reinforcements (including borides, carbides, nitrides, oxides and their mixtures) have undergone fast development [1]. Titanium matrix composites (TMCs) reinforced with silicon carbide $(\mathrm{SiC})$ continuous fibres have a great potential to exploit in the aerospace industry. These composites are stronger, more creep and fatigue resistant and have a lower weight to stiffness ratio than conventional metal alloys [2]. However complex damage and failure behaviours along with prohibitive manufacturing costs and high anisotropic properties are still the main factors that limit their use to highly specialised applications. DMMCs are likely to find high volume of commercial application due to their low cost, ease of fabrication and improved properties. Indeed, by combining metallic properties such as excellent ductility, toughness, formability and good thermal and electric conductivities, with ceramic characteristics, e.g., high hardness, strength, modulus, high-temperature durability and low thermal expansion, the structure is expected to exhibit a higher specific strength, specific stiffness, wear resistance, thermal stability and high-temperature durability compared to the corresponding monolithic matrix materials [3-6]. These superiorities make them potential candidates in the same way as CFMMCs for critical applications in the aerospace and automotive industries. Typical uses of discontinuous or particle titanium composites include creep resistant engineering components, wear parts such as gears, bearings, shafts and erosion-corrosion resistant tubing [7].

Despite highlighted properties and advantages exhibited by particle reinforced titanium matrix composites (PRTMCs), many complex problems that affect the overall mechanical properties and the integrity of the final composite structures still exist. On the one hand, it is necessary to understand the factors that influence the physical and mechanical properties of the composites since they are sensitive to the type of reinforcement, method of manufacture and processing/heat treatment used [8]. On the other hand there is need to carry out an extensive research aimed at providing a better understanding of the cyclic plastic behaviour of TMCs involving creep effects under cyclic thermomechanical loading. Indeed, in the presence of creep, structural response changes substantially under cyclic loading so that lifetime of components can be reduced dramatically.

The representative failure mechanisms associated with the synergistic creep-fatigue interaction are crack initiations due to "low cycle fatigue" (LCF) and "creep ratchetting". LCF induces finite lifetime of components due to high stress levels and a low number of cycles to failure. Common factors that 
have been attributed to creep ratchetting are "cyclic enhanced creep" and "creep enhanced plasticity" $[9,10]$. On the one hand, creep deformation is generally enhanced by cyclic loading especially for longer dwell period. Stress-strain interaction often reports non-closed hysteresis loop due to the enhancement in creep strains, where it is referred to cyclic enhanced creep. On the other hand, if significant stress relaxation occurs within small creep deformation, it can also lead to the creep ratchetting due to large unloading plasticity, where it is referred to the creep enhanced plasticity. To ensure structural integrity, the assessment procedure R5 also recommends to evaluate lifetime of a high temperature component against following mechanisms: plastic collapse failure, creep rupture failure, ratchetting collapse, crack initiation due to creep-fatigue interaction, and excessive cyclic enhanced creep deformation [9]. To satisfy the design requirements, the cyclic plasticity analysis and the cyclic creep and plastic analysis have to be carried out independently. If an applied cyclic loading level is under either strict or global shakedown limit without creep, no ratchetting mechanism appears in the steady state response [11]. However, with creep, for the same loading level applied, ratchetting response can take place due to either cyclically enhanced creep or creep enhanced plasticity depending on primary load level, known as rupture reference stress, or duration of dwell period [1214].

In light of the above considerations this work is focused on the investigation of the both cyclic plasticity and creep-cyclic plasticity behaviours of the PRTMCs by means of a direct numerical method and three dimensional multi-particles unit cells [15-17]. In Section 2, a brief overview of the numerical procedures adopted for this work is provided. Section 3 shows the microstructure generation of the multi-particle unit cells along with the boundary conditions applied for the numerical investigation. Section 4 presents numerical results and discussions. Shakedown limit boundaries for different particle arrangements, number of particles, and particle fractions volume are reported along with variations of the two main critical design criteria i.e. the shakedown limit and the limit load in Section 4.1. In Section 4.2, the creep-cyclic plasticity behaviours of the PRTMCs are presented by hysteresis stress-strain loops constructed in the steady state cycle. The effect of key parameters affecting the cyclic plasticity and creep-cyclic plasticity behaviour of the PRTMCs are comprehensively discussed in subsections of the both Section 4.1 and 4.2. Conclusions and remarks are made in Section 5.

\section{Direct methods employed for the cyclic plastic and cyclic creep and plastic analysis}

To obtain a shakedown limit boundary of the SiC/Ti-6242 PRTMCs subjected to a cyclic thermal load with a constant mechanical load, a numerical direct method called the Linear Matching Method (LMM) is adopted. The LMM matches non-linear material response to a linear material behaviour 
using iterative computational processes by changing the elastic modulus at each integration point of a finite element (FE) model [18]. For the shakedown limit analysis, the LMM computes both upper bound and lower bound limit multipliers under cyclic loadings, creating a load envelope to show a limit of structural responses as Bree like diagram. The LMM was used to analyse the cyclic plasticity of the fibre-reinforced composite materials[19, 20]. The LMM was extended to the Direct Steady Cycle Analysis (LMM DSCA) [21] that calculates the stabilized response of a structure subjected to cyclic loadings with accuracy and efficiency that supersedes other traditional direct methods [22].

The LMM DSCA method was further extended by Chen et al. [23] to evaluate a structural response to creep-cyclic plasticity behaviour in the steady state. The extended Direct Steady Cycle Analysis method (eDSCA) has been actively being utilised to assess the low cycle fatigue, and the creepfatigue damages [24, 25]. A flowchart of the LMM eDSCA procedures is attached in the appendix. Validity and applicability of the LMM framework have been also acknowledged by a variety of commercial industry partners [25-27], in particular the LMM DSCA method has been selected by R5 research programme of EDF energy to the commercial standard [9]. In particular for the composite materials, the eDSCA was employed to analyse the cyclic creep and plastic behaviours [28-30]. Based on the reliability, the LMM eDSCA method is adopted for the numerical analysis in the present work. Brief introductions to the numerical procedures of the adopted methods are made in the following subsection.

\subsection{Numerical procedures for shakedown limit analysis}

It is assumed that a structure follows elastic perfectly plastic model $(E P P)$ with a volume $V$ and a surface area of $S$ as well as satisfying the von-Mises yield condition. The structure is subjected to the cyclic thermal load $\lambda \theta(t)$ acting across the $V$ and the steady mechanical load $\lambda P(t)$ imposing on a part of the surface $S_{T}$ over time period $0 \leq t \leq \Delta t$, where $\lambda$ denotes load parameters. A remaining surface $S_{R}\left(S_{R}=S-S_{T}\right)$ is constrained by no displacement rate $(\dot{U}=0)$. Upon the loading and boundary conditions, a linear elastic stress solution can be expressed by Eq.(1)

$$
\hat{\sigma}_{i j}^{e}(t)=\lambda \hat{\sigma}_{i j}^{\theta}(t)+\lambda \hat{\sigma}_{i j}^{P}(t)
$$

where $\lambda \hat{\sigma}_{i j}^{\theta}$ and $\lambda \hat{\sigma}_{i j}^{P}$ denote changing elastic stresses corresponding to $\theta(t)$ and $P(t)$, respectively. 
For the cyclic problem, a general form of the elastic solution can be expressed by Eq.(2) with three different components; the elastic stress $\hat{\sigma}_{i j}^{e}(t)$, the constant residual stress $\bar{\rho}_{i j}$, and the varying residual stress $\rho_{i j}^{r}(t)$

$$
\hat{\sigma}_{i j}(t)=\hat{\sigma}_{i j}^{e}(t)+\bar{\rho}_{i j}+\rho_{i j}^{r}(t)
$$

The history of $\rho_{i j}^{r}(t)$ is the change in the residual stress within the cycle and satisfies $\rho_{i j}^{r}(0)=\rho_{i j}^{r}(\Delta t)$, so that the stresses and strain rates will become asymptotic to a cyclic state. For the shakedown analysis, $\rho_{i j}^{r}(0)=0$ must be achieved, therefore ratchetting response of the structure will not occur with the zero plastic strain accumulation during the cycles.

The shakedown limit analysis considers a global minimization process to evaluate the $\bar{\rho}_{i j}$ imposed by the combined cyclic and steady loads. The shakedown condition and the global minimization process of the energy based on the Koiter's theorem [31] are integrated, giving a minimization function in an incremental form as Eq.(3),

$$
I\left(\Delta \varepsilon_{i j}, \lambda_{S D}^{U B}\right)=\int_{V} \sum_{n=1}^{N}\left\{\sigma_{i j}^{n} \Delta \varepsilon_{i j}^{n}-\lambda_{S D}^{U B} \hat{\sigma}_{i j}\left(t_{n}\right) \Delta \varepsilon_{i j}^{n}\right\} d V \geq 0
$$

where $\hat{\sigma}_{i j}\left(t_{n}\right)$ is the augmented elastic stress solution with the constant residual stress at a sequence of time $t_{n} ; \Delta \varepsilon_{i j}$ is the strain increment occurring at time $t_{n} ; n$ increases from 1 to $N$ during the cycle; $\lambda_{S D}^{U B}$ is a shakedown upper bound multiplier.

By transforming the Eq. (3) to Eq.(4), the $\lambda_{S D}^{U B}$ can be calculated from

$$
\lambda_{S D}^{U B}=\frac{\int_{0}^{V} \int_{0}^{\Delta t} \sigma_{y} \overline{\dot{\varepsilon}}\left(\dot{\varepsilon}_{i j}\right) d t d V}{\int_{0}^{V} \int_{0}^{\Delta t}\left(\hat{\sigma}_{i j} \dot{\varepsilon}_{i j}\right) d t d V}
$$


where $\sigma_{y}$ is the yield stress; $\hat{\sigma}_{i j}$ is the enhanced elastic stress solution; $\dot{\varepsilon}_{i j}$ is a kinematically admissible strain rate; $\overline{\dot{\varepsilon}}$ is the effective strain rate $\overline{\dot{\varepsilon}}=\sqrt{\frac{2}{3} \dot{\varepsilon}_{i j} \dot{\varepsilon}_{i j}}$.

The upper bound multiplier is updated by the iterative process till converging to the least upper bound limit, satisfying $\lambda_{S D}^{U B} \geq \lambda_{S D}$, where $\lambda_{S D}$ is the exact shakedown limit. The shakedown lower bound multiplier $\lambda_{S D}^{L B}$ is calculated based on Melan's Theorem [32]. By checking the $\rho_{i j}^{r}(t)$ within the computation process of the upper bound multiplier, the iterative process continues to calculate $\lambda_{S D}^{L B}$ until where the modified elastic solution at each integration point does not violate the yield condition of the material, satisfying $\lambda_{S D}^{L B} \leq \lambda_{S D}, \lambda_{S D}^{L B}$ can be expressed by Eq.(5).

$$
f\left(\lambda_{S D}^{L B} \hat{\sigma}_{i j}(t)+\bar{\rho}_{i j}\right) \leq 0
$$

\subsection{Numerical procedures for cyclic creep and plastic analysis}

The eDSCA procedure calculates the cyclic stress history at the steady state in associated with residual stresses accumulated by inelastic strains either plastic or creep during the loading cycle. The eDSCA utilise a similar minimization procedure with Eq.(3) which has an assumption that plastic strain only occurs at time $t_{n}$, where $N$ (from $n=1$ to $N$ ) denotes total number of loading instances. The minimization function of the eDSCA in an incremental form can be given by Eq.(6).

$$
I^{n}\left(\Delta \varepsilon_{i j}^{n}\right)=\int_{V}\left\{\sigma_{i j}^{n} \Delta \varepsilon_{i j}^{n}-\left[\hat{\sigma}_{i j}\left(t_{n}\right)+\rho_{i j}^{r}\left(t_{n}\right)\right] \Delta \varepsilon_{i j}^{n}\right\} d V \geq 0
$$

By an iterative process, the strain increment $\Delta \varepsilon_{i j}^{n}$ can be calculated by the minimization process until the requested a total number of cycles $M$. The number of load instances $N$ is performed as sub-cycles within each cycle $m$, where $m$ (from $m=1$ to $M$ ). Hence, the accumulated residual stress for $\mathrm{n}^{\text {th }}$ load instance at $\mathrm{m}^{\text {th }}$ cycle of iterations can be expressed by Eq.(7). 


$$
\rho_{i j}^{r}\left(t_{n}\right)_{m}=\sum_{i=1}^{m-1} \sum_{n=1}^{N} \Delta \rho_{i j}^{r}\left(t_{n}\right)_{i}+\sum_{i=1}^{n} \Delta \rho_{i j}^{r}\left(t_{i}\right)_{m}
$$

For examples, if the cycles $m$ and $m+1$ are only considered, the iterative shear modulus $\bar{\mu}_{m}\left(t_{n}\right)$ at a load instance $t_{n}$ can be defined by Eq.(8), where $\sigma_{y}\left(t_{n}\right)_{m}$ denotes the von-Mises yield stress of the elastic-perfectly plastic model, which is substituted to creep flow stress $\bar{\sigma}_{c}$ when the $t_{n}$ involves a load instance of creep.

$$
\bar{\mu}_{m+1}\left(t_{n}\right)=\bar{\mu}_{m}\left(t_{n}\right) \frac{\sigma_{y}\left(t_{n}\right)_{m}}{\bar{\sigma}\left(\hat{\sigma}_{i j}\left(t_{n}\right)+\rho_{i j}^{r}\left(t_{n}\right)_{m}\right)}
$$

Without consideration of a load instance of creep, the inelastic strain increment $\Delta \varepsilon_{i j}\left(t_{n}\right)_{m+1}$ at the cycle $m+1$ can be calculated by Eq.(9),

$$
\Delta \varepsilon_{i j}\left(t_{n}\right)_{m+1}^{\prime}=\frac{1}{2 \bar{\mu}_{m}\left(t_{n}\right)}\left\{\hat{\sigma}_{i j}\left(t_{n}\right)+\rho_{i j}^{r}\left(t_{n-1}\right)_{m+1}+\Delta \rho_{i j}^{r}\left(t_{n}\right)_{m+1}\right\}^{\prime}
$$

where $\rho_{i j}^{r}\left(t_{n-1}\right)$ is the accumulated previous residual stress before the time $t_{n}$ and the notation (') refers to the deviatoric component.

For creep dwell, an effective creep strain $\Delta \bar{\varepsilon}^{c}$ is calculated using time hardening power law known as Bailey-Norton law as given Eq.(10), where $A, n^{*}, m^{*}$ are temperature dependent material properties for creep behaviour and $\Delta t$ denote time for creep dwell. $\bar{\sigma}_{s}$ and $\bar{\sigma}_{c}$ are the start of creep stress and the creep flow stress at the end of creep dwell respectively. The creep strain rate $\dot{\bar{\varepsilon}}^{c}$ at the dwell time $\Delta t$ is calculated by Eq.(11). 


$$
\begin{gathered}
\Delta \bar{\varepsilon}^{c}=\frac{A\left(n^{*}-1\right) \Delta t^{m^{*}+1}\left(\bar{\sigma}_{s}-\bar{\sigma}_{c}\right)}{\left(\bar{\sigma}_{c}^{-n^{*}+1}-\bar{\sigma}_{s}^{-n^{*}+1}\right)\left(m^{*}+1\right)} \\
\dot{\bar{\varepsilon}}^{c}=\frac{\Delta \bar{\varepsilon}^{c}\left(m^{*}+1\right) \bar{\sigma}_{c}^{n^{*}}}{\left(n^{*}-1\right)\left(\bar{\sigma}_{s}-\bar{\sigma}_{c}\right) \Delta t}\left(\bar{\sigma}_{c}^{-n^{*}+1}-\bar{\sigma}_{s}^{-n^{*}+1}\right)
\end{gathered}
$$

Initially, the iterative process starts with estimated $\bar{\sigma}_{s}$ and $\bar{\sigma}_{c}$ values. The equations (10) and (11) compute new creep flow stress $\bar{\sigma}_{c}^{f}$ using Eq.(12), so that the $\bar{\sigma}_{c}{ }^{f}$ replace $\sigma_{y}\left(t_{n}\right)_{m}$ in Eq.(8) to carry out the linear matching condition.

$$
\bar{\sigma}_{c}^{f}=\left(\frac{\dot{\bar{\varepsilon}}^{c}}{A \Delta t^{m^{*}}}\right)^{\frac{1}{n^{*}}}
$$

\section{Problem description}

\subsection{Microstructure generation and boundary conditions}

In order to carry out this numerical investigation, three-dimensional multi-particle unit cells have been employed. The FE models used in this paper have been taken from a previous study on the optimization of the particle spatial distribution of MMCs [33]. The main finding of [33] was the development of a Low-Fidelity Model-Based Optimization (LFMBO) aiming at maximising the uniaxial Young's modulus by varying the particle spatial distribution. RVEs characterised by both different number of particles (from 1 to 20 ) and particle volume fractions (from $1 \%$ to $25 \%$ ) have been investigated.

The optimization method relies upon the coupling between Matlab Global Optimization Toolbox [34], Python [35] and Abaqus FEA [36] as summarised in Figure 1. The optimization starts with a repaired Latin Hypercube design of experiment [37] and the most promising candidates are selected to constitute the population of first generation (set of green boxes in Figure 1). Then, the optimisation loop starts (set of red boxes in Figure 1). The objective and the constraints violation are computed for each candidate and, in light of these, a fitness value is assigned. Next, the candidates are ranked and the most promising are selected and used by the Genetic Algorithms operators, namely elitism, crossover and mutation [38], to generate the population of the next generation.

Afterwards, with a probability of 5\%, a Monotonic Basin Hopping (MBH) [39] based optimization is performed starting from the current best candidate.

The algorithm's control parameters and the stopping criteria are not fixed but rather change in accordance of the progress of the optimisation as described in [33]. 
The optimization process continues restarting the loop from the proposed next population until the stopping criteria are met. Once the optimization is reputed terminated, the uniaxial Young's modulus resulting from the optimum particle spatial distribution found is computed through a high-fidelity procedure. This is composed by coupling different software modules, i.e., Matlab Objective function Code and a set of Python scripts for Abaqus FEA which is comprised of RVE generator, PBC adaptive code, pre-processing code and homogenization code (set of blue boxes in Figure 1). The objective function works as wrapper function to interface GA to the RVE Generator code. The latter generates the FE models according to the optimization variables while the pre-processing code automatically assigns the materials properties, generates the mesh and runs the PBC code. This assigns the periodic boundary conditions [40] according to the equations (13), (14), and (15), to all the relative node pairs on the boundary faces i.e. inner face nodes, inner edge nodes and corner nodes as depicted in Figure 2. Into the aforementioned equations, $u_{i}$ is the nodal variable at a specific node within a node group related to the degree of freedom $i$ while $u_{i}^{D_{1}}, u_{i}^{D_{2}}$, and $u_{i}^{D 3}$ are the perturbation carried out on the dummy nodes $D_{1} D_{2}$ and $D_{3}$ and $\mathrm{L}$ is the length of the RVE's edge. The perturbations imposed are $u_{i}^{D_{1}} \neq 0$ and $u_{i}^{D_{2}}=u_{i}^{D 3}=0$. Finally, the FE analysis is performed and from the generated ODB file the homogenised uniaxial Young' modulus is computed. Hence, the outcome of the research is a set of RVEs coming from the optimization process for all the investigated number of particles and fraction volumes.

These arrays have been used for the simulation model setup of the current study because they allow to investigate the effect of particle spatial distribution as well as particle volume fraction and particle inter-spacing due to the variety of arrangements that the optimiser has found. 

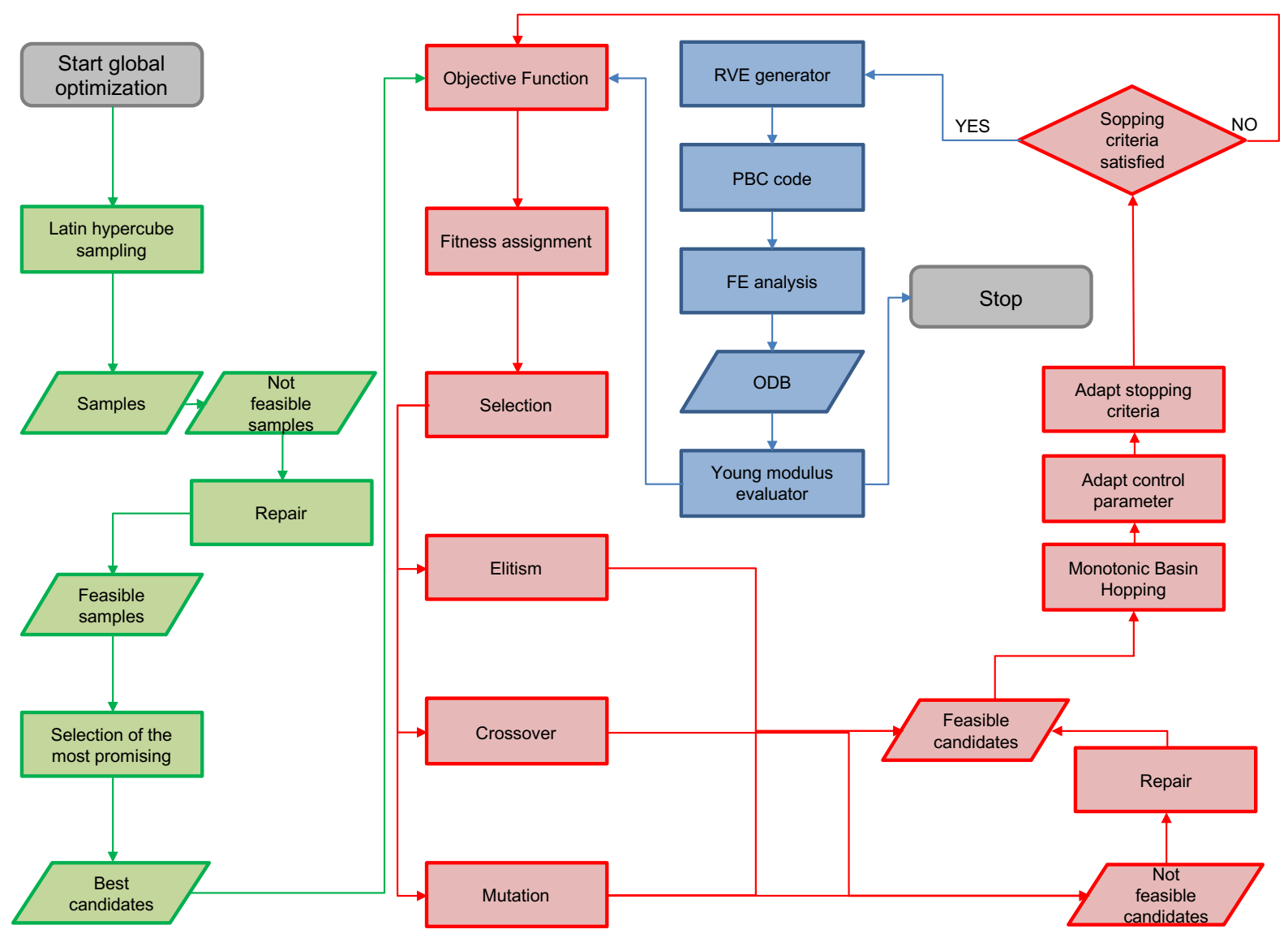

Figure 1 Low fidelity model-based optimization flow chart.

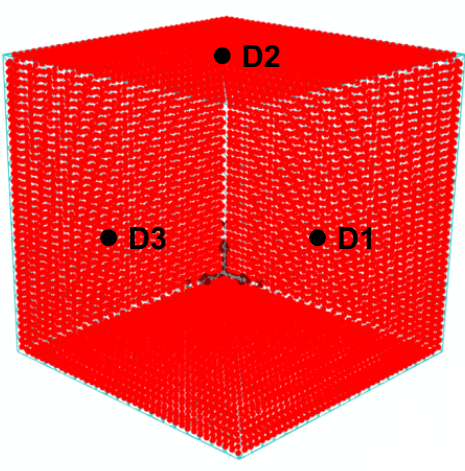

a)

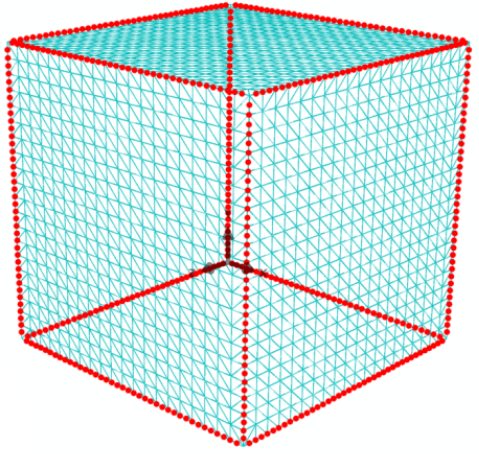

b)

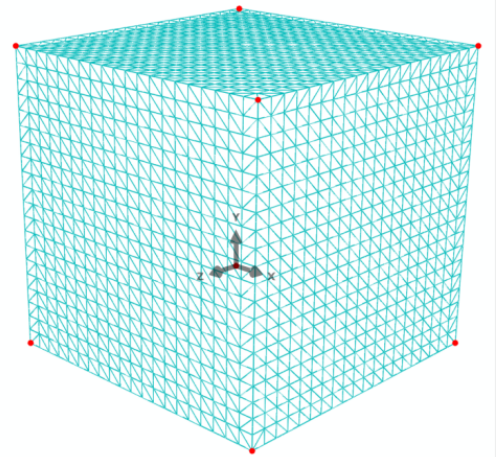

c)

Figure 2 Groups of nodes on the boundary faces of the RVEs a) inner face nodes, b) inner edge nodes, c) corner nodes. 


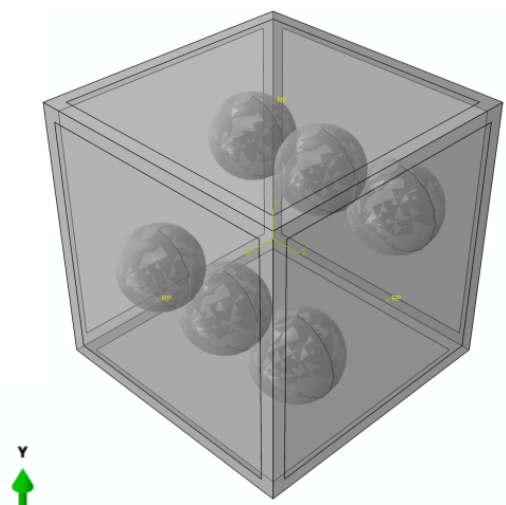

a)

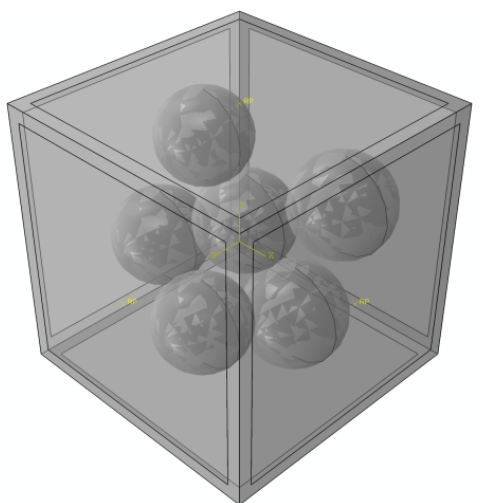

b)

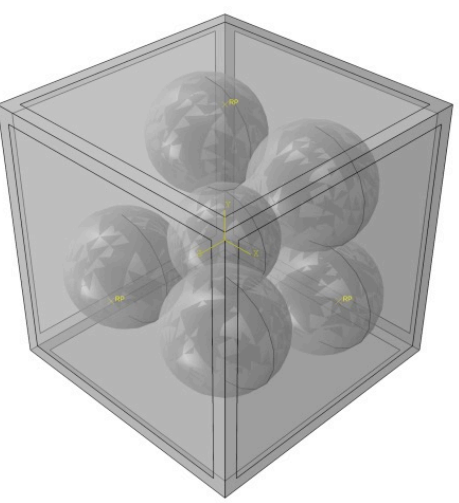

c)

Figure 3 Typical FE models with six particles and a) $V_{f}=10 \%$, b) $V_{f}=14 \%$, c) $V_{f}=25 \%$.

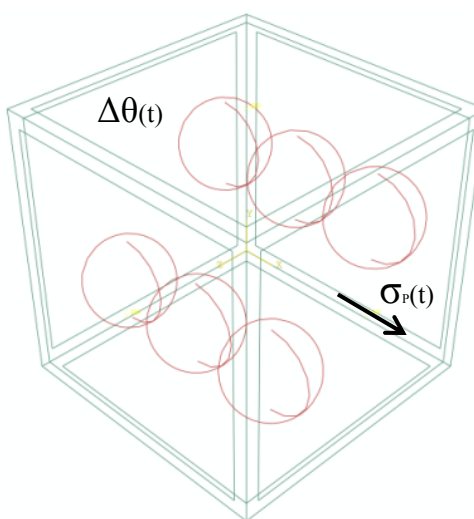

a)

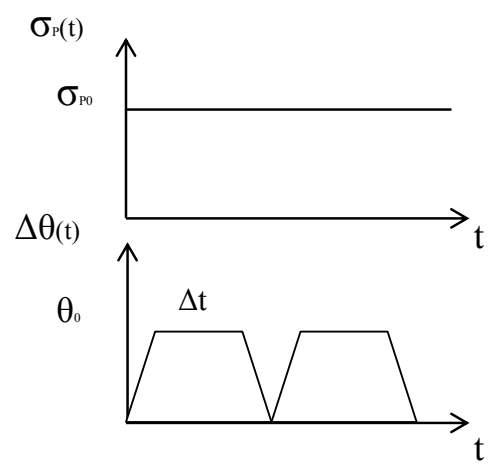

b)

Figure 4 a) RVE with tensile mechanical load $\sigma_{p}(t)$ and cyclic thermal load $\Delta \theta(t)$, b) load history applied.

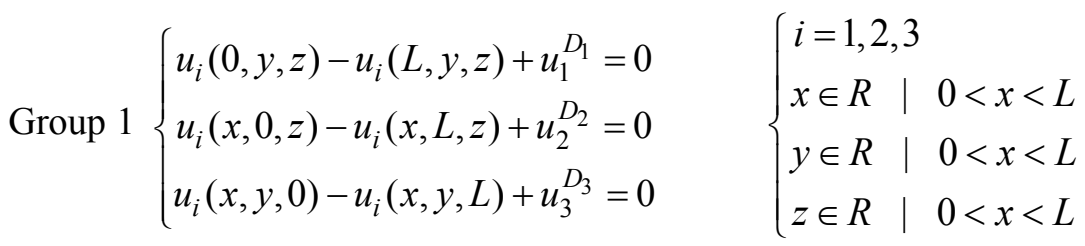




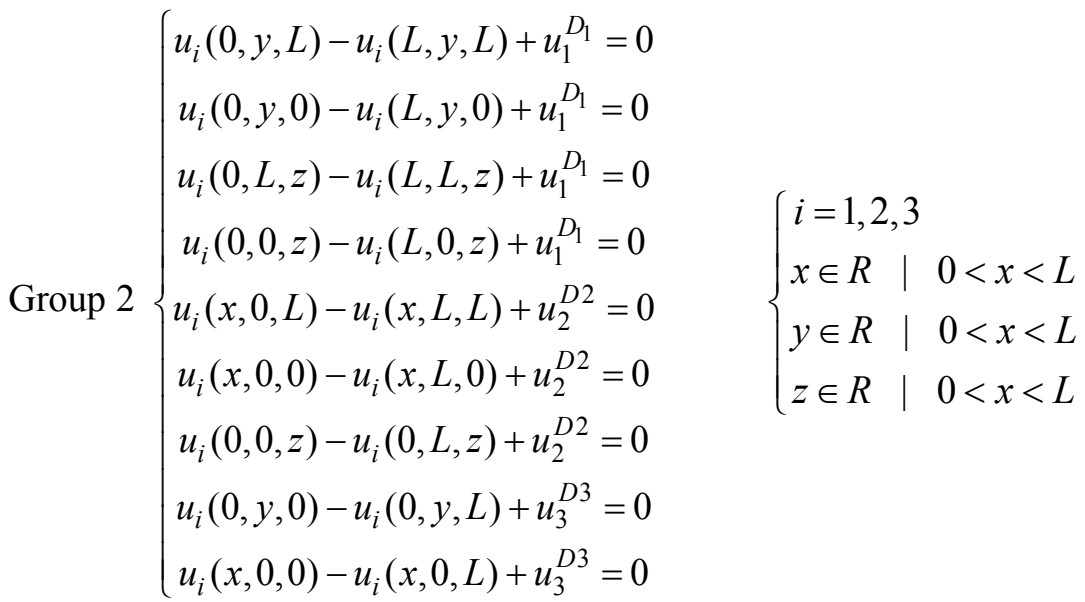

Group $3\left\{\begin{array}{l}u_{i}(0, L, L)-u_{i}(L, L, L)+u_{1}^{D 1}=0 \\ u_{i}(0,0, L)-u_{i}(L, 0, L)+u_{1}^{D 1}=0 \\ u_{i}(0, L, 0)-u_{i}(L, L, 0)+u_{1}^{D 1}=0 \\ u_{i}(0,0,0)-u_{i}(L, 0,0)+u_{1}^{D 1}=0 \\ u_{i}(0,0, L)-u_{i}(0, L, L)+u_{2}^{D 2}=0 \\ u_{i}(0,0,0)-u_{i}(0, L, 0)+u_{2}^{D 2}=0 \\ u_{i}(0,0,0)-u_{i}(0,0, L)+u_{3}^{D 3}=0\end{array} \quad\{i=1,2,3\right.$

\subsection{Simulation model setup}

Among the optimized arrays of [33], we have considered three fraction volumes of the reinforcement hereinafter referred to as $\mathrm{V}_{\mathrm{f}}$, i.e. $\mathrm{V}_{\mathrm{f}}=10 \%, \mathrm{~V}_{\mathrm{f}}=14 \%, \mathrm{~V}_{\mathrm{f}}=25 \%$, and five particle distributions with 2, 4, 6,8 , and 10 number of particles hereinafter referred to as $\mathrm{N}_{\text {part }}$. Three of the FE models employed are shown in Figure 3 and a typical RVE is meshed with Abaqus C3D10 tetrahedral and is comprised of roughly 60,000 elements which is the best compromise, found by a preliminary mesh convergence study $[33,44]$, in terms of accuracy and computational time. The effect of particle spatial distribution, number of particles, and particle volume fractions on the shakedown behaviour has been investigated by superimposing a tensile mechanical load $\sigma_{p}(t)$ along the $x$ direction on a cyclic thermal load $\Delta \theta(t)$ with a dwell time $\Delta \mathrm{t}=0$ (Figure 4-b). The shakedown response has been the starting point for the investigation of the creep-fatigue interaction response where different dwell times have been considered i.e. $\Delta \mathrm{t}=1 \mathrm{hr}, 10 \mathrm{hrs}$, and $100 \mathrm{hrs}$. When a dwell time is introduced within the thermal load history, different mechanisms can arise depending upon the loading condition applied and the state of stress at the matrix-reinforcement interface [41]. Indeed as reported in [24] within the shakedown boundary the structure can exhibit either shakedown or creep enhanced plasticity. The latter can lead to two different scenarios which are closed loop or creep-ratchetting. Instead, beyond the shakedown boundary, creep-ratchetting is expected in most cases, which is a dangerous mechanism that has to be avoided. 
Figure 4 shows a typical RVE with the mechanical load applied on the dummy node $D_{l}$ and the thermal load applied by Abaqus temperature field throughout its region. The former load is constant in time while the latter varies in time as shown in Figure 4-b.

Constituent material properties were chosen to correspond to elastic SiC particles perfectly bonded to a Ti-6242 matrix that follow the data reported in Table 1. Due to the high temperature, it is relevant to evaluate the creep strain only for the titanium matrix. The creep constitutive equation adopted is the Norton law that represents the steady-state creep rate of the material within the secondary creep stage:

$$
\dot{\varepsilon}^{c}=A \cdot \sigma^{n} \cdot \exp (-Q / R T)
$$

where $\dot{\varepsilon}^{c}$ denotes creep strain rate, $\mathrm{n}$ is the stress exponent, $\mathrm{A}$ is a constant, $\mathrm{Q}$ is the activation energy, $\mathrm{R}$ is the universal constant of gases, and $\mathrm{T}$ is the absolute temperature $[\mathrm{K}]$.

Table 1 Mechanical properties at $500^{\circ} \mathrm{C}$.

\begin{tabular}{lcc}
\hline & $\mathrm{SiC}$ & $\mathrm{Ti}-6242$ \\
\hline $\mathrm{E}[\mathrm{GPa}]$ & 380 & 95 \\
\hline$v$ & 0.19 & 0.32 \\
\hline$\alpha\left[{ }^{\circ} \mathrm{C}^{-1}\right]$ & $4.1 \mathrm{e}^{-6}$ & $8.1 \mathrm{e}^{-6}$ \\
\hline$\sigma_{\mathrm{y}}[\mathrm{MPa}]$ & 3450 & 350 \\
\hline
\end{tabular}

Creep parameters are taken from the tensile creep test data of Ti-6Al-4V material in which test performed at $200 \mathrm{MPa}$ and $600^{\circ} \mathrm{C}$ [42]. Ti-6Al-4V material shows similar creep behaviour with Ti6242. The creep properties are summarized in Table 2.

Table 2 Creep parameters.

\begin{tabular}{ccccc}
\hline Material & $\mathrm{A}\left[\mathrm{MPa}^{-n} h^{-1}\right]$ & $\mathrm{n}$ & $\mathrm{Q}\left[\mathrm{kJ} \cdot \mathrm{mol}^{-1}\right]$ & $\mathrm{R}\left[\mathrm{J} \cdot \mathrm{mol}^{-1} \cdot \mathrm{K}^{-1}\right]$ \\
\hline Ti-6Al-4V & 4432.45 & 4.6 & 267 & 8.314 \\
\hline
\end{tabular}




\section{Results and discussion}

4.1. Shakedown behaviour and load bearing capacity of a SiC-Ti6242 PRTMCs at $500^{\circ} \mathrm{C}$

Shakedown boundaries, reverse plasticity limits $\Delta \theta_{r p}$ as well as limit loads $\sigma_{p l}$ are reported in the form of interaction diagram for all the RVEs investigated. $\Delta \theta_{r p}$ is the maximum of the thermal load range $\Delta \theta$ related to a specific RVE's configuration characterized by $\mathrm{N}_{\text {part }}$ and $\mathrm{V}_{\mathrm{f}}$, beyond which reverse plasticity occurs while $\sigma_{p l}$ is the maximum load for a specific RVE's configuration that the structure can safely carry [19]. For the shakedown limit boundaries the axes are expressed in nondimensional variables $\Delta \theta / \Delta \theta_{0}$ and $\sigma_{p} / \sigma_{p_{0}}$ where $\Delta \theta_{0}=500^{\circ} \mathrm{C}$ is the reference thermal load range whilst $\sigma_{p_{0}}=350 M P a$ is the matrix yield stress. The three loads points $\mathrm{P} 1\left(\sigma_{p}=0\right.$ and $\left.\theta=0.8 \theta_{0}\right)$, P2 $\left(\sigma_{p}=0.15 \sigma_{p 0}\right.$ and $\left.\theta=0.8 \theta_{0}\right), \mathrm{P} 3\left(\sigma_{p}=0.3 \sigma_{p 0}\right.$ and $\left.\theta=0.8 \theta_{0}\right)$ are examined for different dwell times in order to investigate the creep-cyclic plasticity behaviour within the shakedown zone for all particle arrangements studied.

\subsubsection{Effect of number of particles, particle fraction volume and particle} arrangement on the shakedown boundaries

Shakedown boundaries and the variation of the critical design limits i.e shakedown limit $\Delta \theta_{r p}$ and limit load $\sigma_{p l}$ for different $\mathrm{N}_{\text {part }}$ i.e. $2,4,6,8,10$ and different $\mathrm{V}_{\mathrm{f}}$ i.e. $10 \%, 14 \%$, and $25 \%$, are plotted in Figure 5 and Figure 7 respectively. 


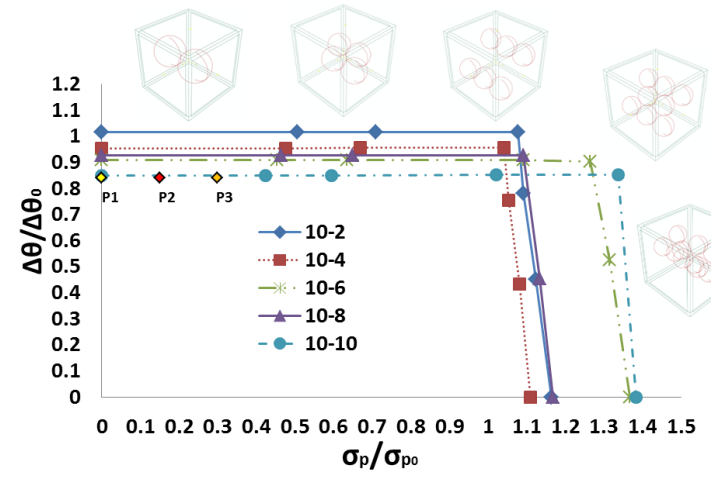

a)

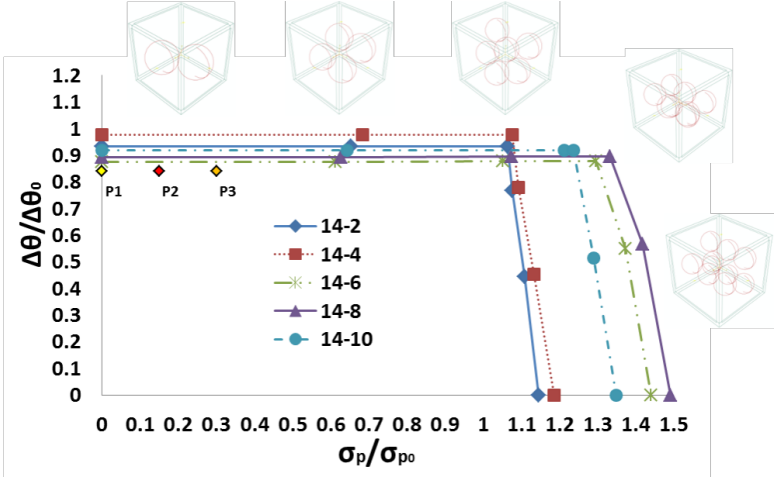

b)

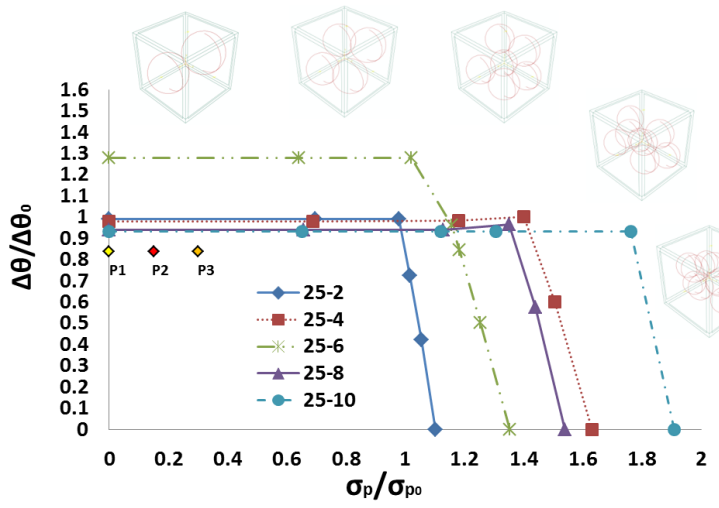

c)

Figure 5 Shakedown boundaries for different particle arrangements with $N_{\text {part }} 2,4,6,8$, and 10 and a) $V f=10 \%$, b) $V f=14 \%, c) V f=25 \%$.

Along with the variables $\mathrm{N}_{\text {part }}$ and $\mathrm{V}_{\mathrm{f}}$, the particle spatial distribution influences both the shakedown behaviour and the critical design limits. Indeed, by comparing the 5 arrays for the three fraction volumes investigated there is not a clear trend as for $\mathrm{V}_{\mathrm{f}}=10 \%$ (Figure 5-a) the array with $\mathrm{N}_{\text {part }}=2$ has the highest $\Delta \theta_{r p}$ whilst for $\mathrm{V}_{\mathrm{f}}=14 \%$ (Figure 5-b) and $\mathrm{V}_{\mathrm{f}}=25 \%$ (Figure 5-c) the highest $\Delta \theta_{r p}$ is computed for $\mathrm{N}_{\text {part }}=4$ and $\mathrm{N}_{\text {part }}=6$ respectively. A different scenario is seen for the limit load where for $\mathrm{V}_{\mathrm{f}}=10 \%$ and $\mathrm{V}_{\mathrm{f}}=25 \%$ the highest $\sigma_{p l}$ is computed for $\mathrm{N}_{\text {part }}=10$ whilst for $\mathrm{V}_{\mathrm{f}}=14 \%$ the highest $\sigma_{p l}$ is computed for $\mathrm{N}_{\text {part }}=8$. This explains the effect of the particle spatial distribution on the shakedown behaviour which, as reported in other studies [33, 43, 44] must be taken into account as it strongly affects the elastoplastic behaviour of composites. Also, as can be seen from Figure 6 the particle spatial distribution influences the thermo-elastic stress which directly affects the reverse plasticity limit $\Delta \theta_{r p}$. Indeed, by comparing the three different particle arrangements for $\mathrm{N}_{\text {part }}=2$ (upper row) the highest thermo-elastic stress computed for $\mathrm{V}_{\mathrm{f}=14 \%}$ leads to a lower $\Delta \theta_{r p}$ compared to $\mathrm{V}_{\mathrm{f}}=10 \%$ and $\mathrm{V}_{\mathrm{f}}=25 \%$. Same scenario can be seen for $\mathrm{N}_{\text {part }}=6$ where the highest $\Delta \theta_{r p}$ is computed for $\mathrm{V}_{\mathrm{f}}=25 \%$ whereby the thermo-elastic stress computed is lower than $V_{f}=10 \%$ and $V_{f}=14 \%$ (Figure 7-a). 


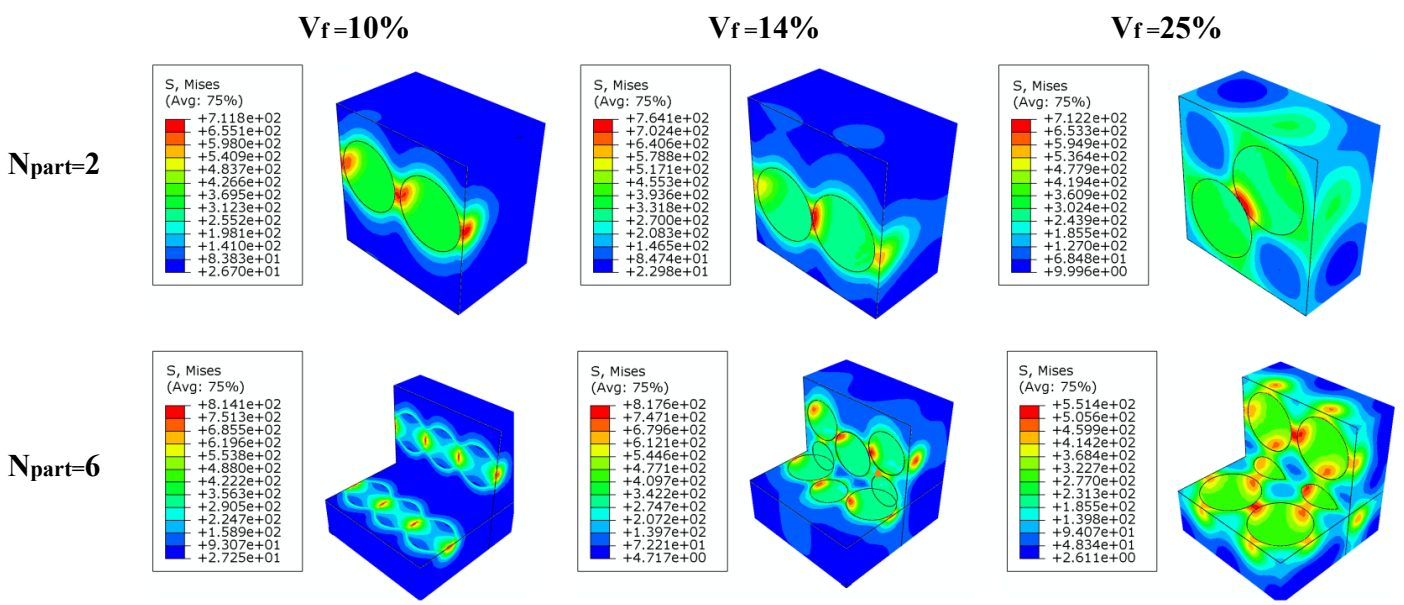

Figure 6 Thermo-elastic stress under $\Delta \theta_{0}=500^{\circ} \mathrm{C}$ for the three different particle arrangements with $N_{\text {part }}=2$ (upper row) and with $N_{\text {part }}=6$ (lower row) for the three different fraction volumes investigated.

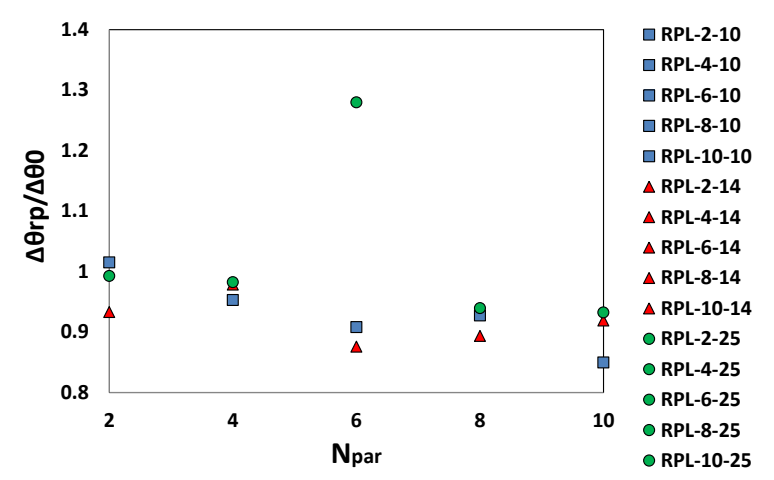

a)

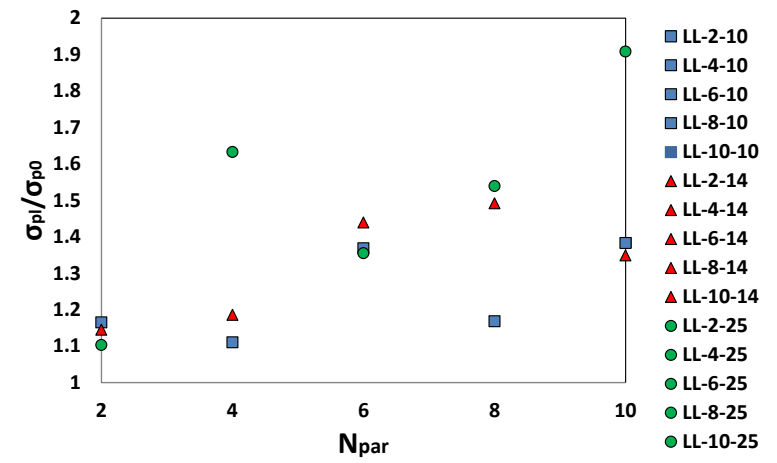

b)

Figure 7 Variation of the critical design limits for different particle arrangements with Npart (2, $4,6,8,10)$ and $V f(10 \%, 14 \%, 25 \%)$ a) reverse plasticity limit, b) limit load.

With regards to the limit load $\sigma_{p l}$, reported in Figure 7-b, the variation for different particles arrangements is mainly influenced by the particle fraction volume as for a higher volume of the reinforcement the matrix carries a lower percentage of the load applied. However the particle spatial distribution can influence the particle load carrying capacity $[45,46]$ as shown for $\mathrm{N}_{\text {part }}=6$ in Figure 7b. Indeed, by comparing $\mathrm{N}_{\text {part }}=6$ with $\mathrm{N}_{\text {part }}=10$ for the three fraction volumes investigated, it can be seen that for $\mathrm{N}_{\text {part }}=6$ the highest limit load is computed for $\mathrm{V}_{\mathrm{f}}=14 \%$ and the lowest limit load for $\mathrm{V}_{\mathrm{f}}$ $=25 \%$ while for $\mathrm{N}_{\text {part }}=10$ the highest limit load is computed for $\mathrm{V}_{\mathrm{f}}=25 \%$ and the lowest limit load for $V_{f}=14 \%$. This means that the particle distribution of the arrangement $N_{\text {part }}=6$ with $V_{f}=14 \%$ leads to a higher limit load than the arrangement $\mathrm{N}_{\text {part }}=6$ with $\mathrm{V}_{\mathrm{f}}=25 \%$. Hence the particle spatial distribution predominates over the fraction volume as under the limit load the particles of the arrangement $\mathrm{N}_{\text {part }}=6$ with $V_{f}=14 \%$ carries a higher load than the particles of the arrangement $N_{\text {part }}=6$ with $V_{f}=25 \%$ (Figure 
8). A different scenario is seen in Figure 8 for $\mathrm{N}_{\text {part }}=10$ where the fraction volume of the reinforcement predominates over the particle spatial distribution.

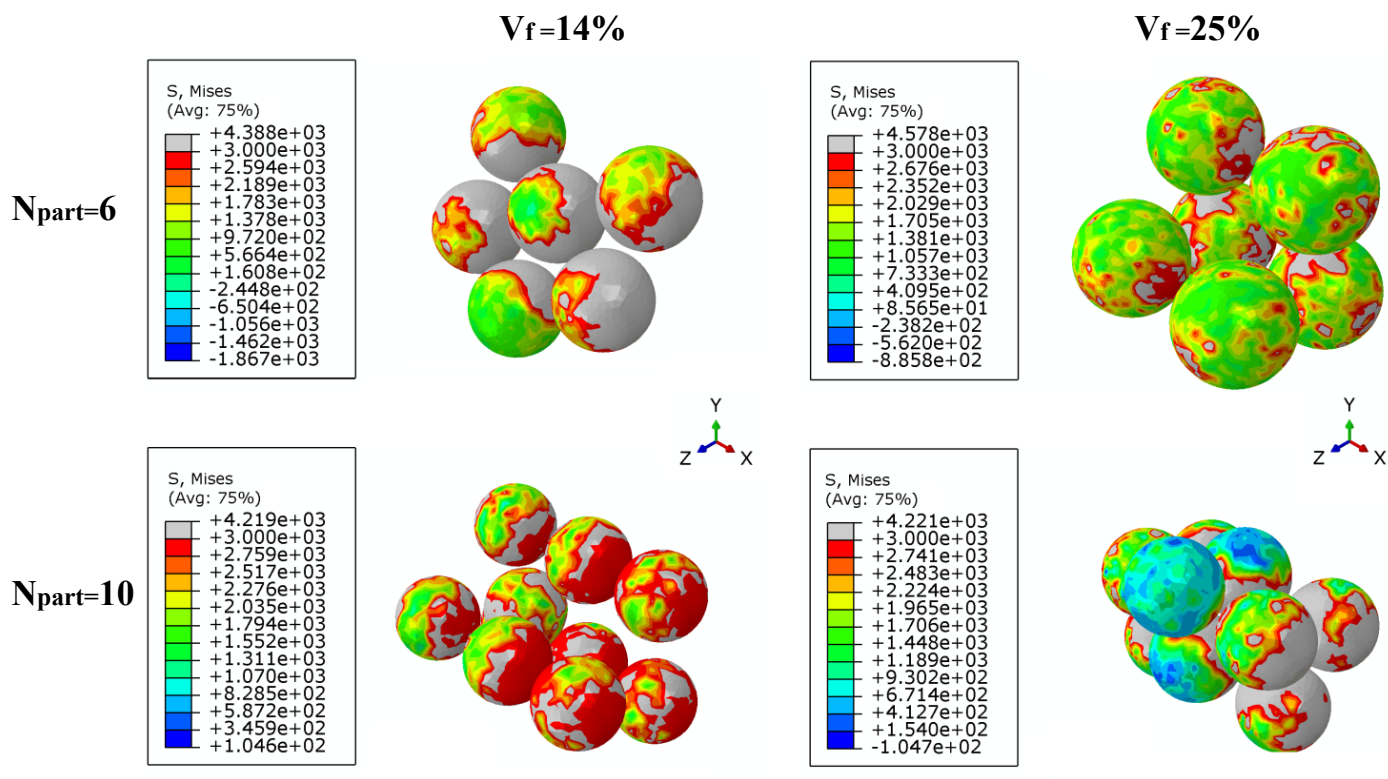

Figure 8 Mises stress distribution under the limit load $\sigma_{p l}$ for four different arrangements with $N_{\text {part }}=6$ and $N_{\text {part }}=10$ for $V_{f}=14 \%$ and $V_{f}=25 \%$.

\subsubsection{Variation of the critical design limits for a fixed particle} arrangement

Figure 9 shows the variation of $\Delta \theta_{r p}$ and $\sigma_{p l}$ with $\mathrm{V}_{\mathrm{f}}$ for two fixed particle arrangements and two different number of particles i.e. the arrangements for $\mathrm{V}_{\mathrm{f}}=25 \%$ with $\mathrm{N}_{\text {part }}=6$ and $\mathrm{N}_{\text {part }}=10$. The results show that the reverse plasticity limit decreases with $V_{f}($ Figure 9-a) while the limit load increases with $\mathrm{V}_{\mathrm{f}}$ (Figure 9-b). These expected findings clarify some aspect already discussed in the previous subsection. Indeed, for the reverse plasticity limit, by increasing the fraction volume for a fixed arrangement the thermo-elastic stress at the particle/matrix interface is expected to increase leading to a lower $\Delta \theta_{r p}$. Instead, the limit load increases with $\mathrm{V}_{\mathrm{f}}$ for a fixed arrangement due to the capability of the reinforcement to carry a higher percentage of the load applied. It is also worth noting that the array with the lower number of particles experiences a higher reverse plasticity limit and a lower limit load throughout the fraction volume range investigated.

The importance of the shakedown boundaries in the prediction of the key parameters for the creepfatigue interaction behaviour will be clarified in the next sections. Even though the three load points investigated i.e. P1, P2, and P3, are within the shakedown zone as shown in Figure 5 the value of the 
creep strain computed as well as the mechanism experienced by the structure will be affected by the value of the reverse plasticity limit.

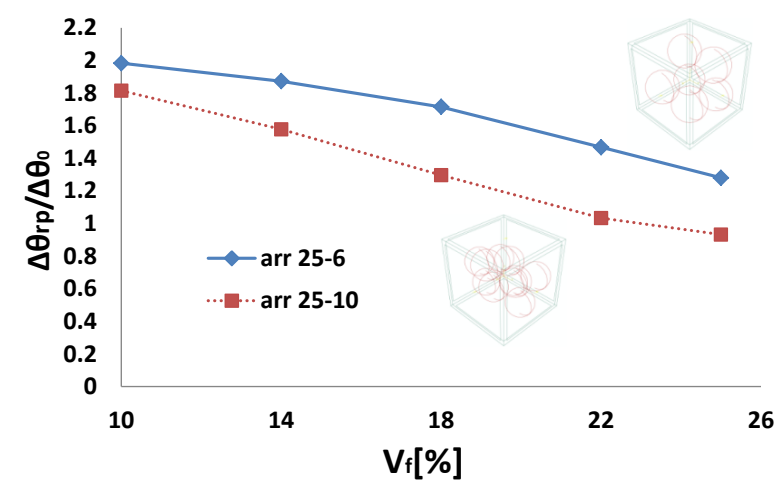

a)

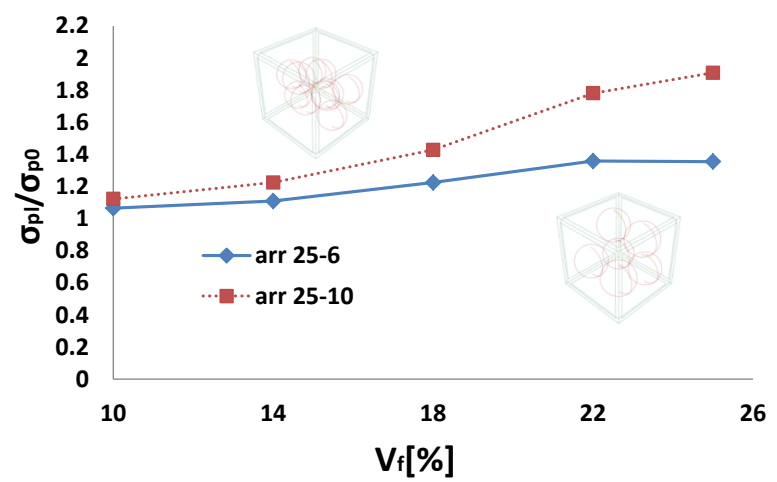

b)

Figure 9 a)Variation of the reverse plasticity limit with $V_{f}$ for two fixed arrangements;

b)Variation of the limit load with $V_{f}$ for two fixed arrangements;

\subsection{Creep-cyclic plasticity behaviour of SiC-Ti6242 PRTMCs}

Cyclic creep behaviours of the PRTMCs subjected to different cyclic load points which are P1 $\left(\sigma_{p}=0\right.$ and $\left.\theta=0.8 \theta_{0}\right), \mathrm{P} 2\left(\sigma_{p}=0.15 \sigma_{p 0}\right.$ and $\left.\theta=0.8 \theta_{0}\right), \mathrm{P} 3\left(\sigma_{p}=0.3 \sigma_{p 0}\right.$ and $\left.\theta=0.8 \theta_{0}\right)$ are analysed by means of the LMM eDSCA method. Eq.(16 is used to obtain the creep parameter for the thermal loading $\theta=0.8 \theta_{0}$. A full incremental cyclic analysis is performed to verify the results analysed from the LMM eDSCA using Abaqus step-by-step (SBS) method for an RVE with $\mathrm{N}_{\text {part }}=6$ and $\mathrm{V}_{\mathrm{f}}=10 \%$ subjected to the load point $\mathrm{P} 2$.

Figure 10 presents resultant creep strain increment in the steady state cycle analysed from the SBS (Figure 10-a) and the LMM eDSCA (Figure 10-b), where, the legends in Figure 10, SDV6 denotes creep strain increment computed by the LMM eDSCA, CEMAG indicates creep strain magnitude analysed by SBS method. The creep strain computed by the LMM eDSCA is in line with the result by the SBS. It is worth noting that the LMM eDCSA produces the reliable results within short computational time less than $10 \%$ of the SBS. Based on the efficient performance, the LMM eDSCA has been selected to analyse the creep-cyclic plasticity response of the PRTMCs rather than the SBS method. 


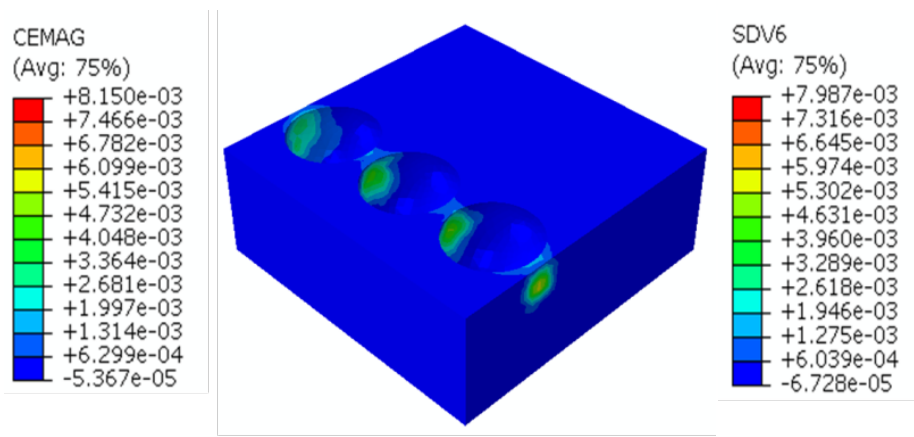

a)

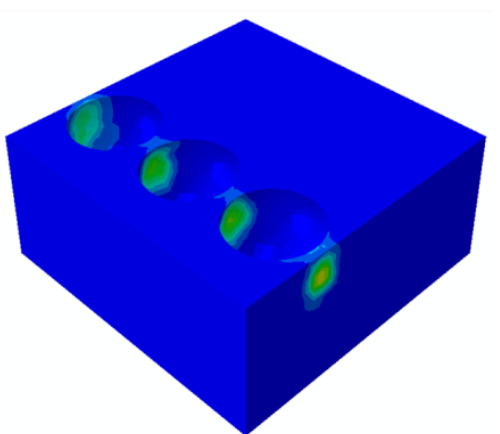

b)

Figure 10 Creep strain contours for the micro scaled SiC-Ti6242 PRTMCs subjected to a cyclic load point $\mathrm{P} 2$ for dwell time of 10hrs; analysed by a) Abaqus step-by-step method and b) the

\section{LMM eDSCA.}

\subsubsection{Effect of particle spatial distribution on the creep-cyclic plasticity response}

The fifteen different RVEs subjected to a cyclic load point P2 in Figure 5 are analysed for a dwell time of $10 \mathrm{hrs}$. Creep strain and ratchetting strain calculated for the most critical location where each RVE model has the highest creep strain accumulated. The results are summarized with respect to a different fraction volume in Table 3. Stress-strain hysteresis loops for notable RVE models that have significant creep strain or ratchetting strain are presented in Figure 11.

Table 3 Creep strain and ratchetting strain from the cyclic creep and plastic analyses of all the RVE models.

\begin{tabular}{ccccccc}
\hline & \multicolumn{5}{c}{ Fraction volume } \\
\hline \multirow{2}{*}{$\begin{array}{c}\text { No. of } \\
\text { particles }\end{array}$} & \multicolumn{2}{c}{$10 \%$} & \multicolumn{2}{c}{$14 \%$} & \multicolumn{2}{c}{$25 \%$} \\
\cline { 2 - 7 } & Creep & Ratchetting & Creep & Ratchetting & Creep & Ratchetting \\
strain (\%) & strain (\%) & strain (\%) & strain (\%) & strain (\%) & strain (\%) \\
\hline 2 & 0.57 & 0.28 & 0.55 & 0.25 & 1.92 & 2.00 \\
4 & 0.52 & 0.30 & 0.46 & 0.23 & 0.38 & 0.16 \\
6 & 0.62 & 0.18 & 0.70 & 0.43 & 0.15 & 0.07 \\
8 & 0.51 & 0.24 & 0.69 & 0.22 & 0.38 & 0.10 \\
10 & 1.65 & 0.32 & 0.42 & 0.10 & 0.51 & 0.09 \\
\hline
\end{tabular}




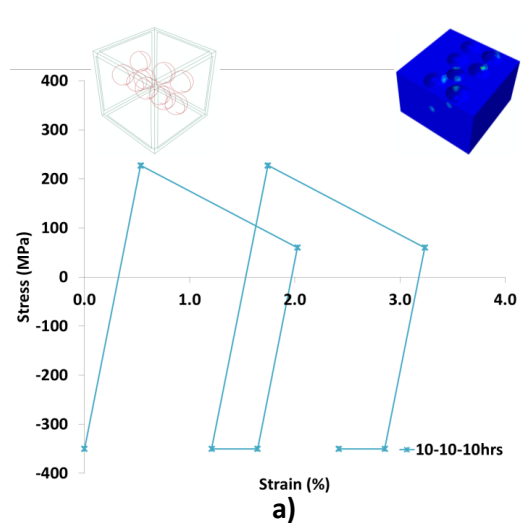

Strain $(\%)$
a)

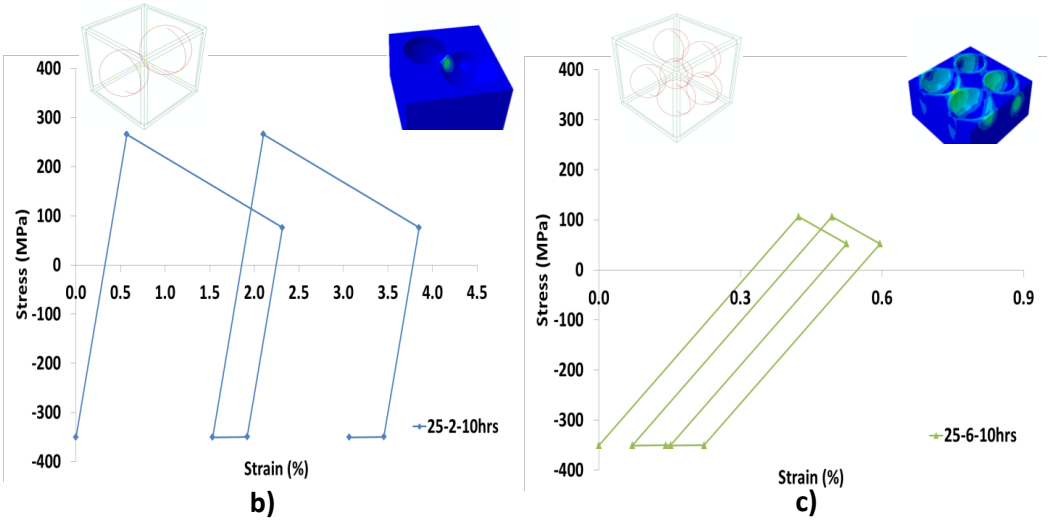

b)

c)

Figure 11 Steady state hysteresis loops of the RVE models subject to cyclic loading P2 for dwell time 10hrs; a) $N_{p a r t}=10$ with $V_{f}=10 \%$, b) $N_{p a r t}=2$ with $V_{f}=25 \%$, and c) $N_{p a r t}=6$ with $V_{f}=25 \%$.

Investigation results in [24] demonstrate that when a structure is subjected to a cyclic loading point under shakedown boundary, structural responses in the steady state are likely to appear either no plastic strain increment or the creep enhanced plasticity. In this work, however, despite the loading level P2 located under the shakedown boundary for all RVEs, it is observed that all RVEs experience creep ratchetting by the cyclically enhanced creep. As mentioned, without creep effects, cyclic loading under both elastic and global shakedown limits do not induce any ratchetting mechanism. However, with creep, the structural response can be the creep ratchetting due to a variety of factors such as geometry, creep constant, other material properties. Hence the creep-cyclic plasticity behaviours of a structure should not be predefined by the cyclic loading level but should be thoroughly investigated. From the results, we have seen no clear correlations between either creep strain or ratchetting strain and the number of particles for a fixed volume fraction. Therefore, the results demonstrate that spatial particle distributions have significant effects on high temperature damage tolerance.

From the results we have seen no clear correlations of either creep strain or ratchetting strain with the number of particles for a fixed fraction volume. It is worth noting that, as explained in section 4.1.1, the particle spatial distribution of the PRTMCs used in the present work has mainly influence on the elastoplastic behaviour of composite materials. Therefore, it can be understood that each RVE exhibits independent creep strain and ratchetting strain increments in the steady state.

Some remarkable results are presented in Figure 11. The RVE for $\mathrm{N}_{\text {part }}=10$ with $\mathrm{V}_{\mathrm{f}}=10 \%$ and another RVE for $\mathrm{N}_{\text {part }}=2$ with $\mathrm{V}_{\mathrm{f}}=25 \%$ show the largest creep deformation. In particular, the latter RVE develops significant total strain range over $2 \%$ within a cycle. Referring to the cyclic loading P2 of the two concerning RVEs in Figure 5, their loading levels are quite close to the reverse plasticity limit $\Delta \theta_{r p}$. On the contrary, RVE for $\mathrm{N}_{\text {part }}=6$ with $\mathrm{V}_{\mathrm{f}}=25 \%$ shows the highest creep endurance under the 
same loading P2, where the level is the furthest down from its reverse plasticity limit. Based on these observations, we can see that the cyclic load points closer to reverse plasticity limit may cause significant creep deformation.

\subsubsection{Effect of varying dwell time and tensile load level on the creep- cyclic plasticity response}

The parametric studies are carried out for an RVE model which has $\mathrm{N}_{\text {part }}=6$ with $\mathrm{V}_{\mathrm{f}}=10 \%$ in order to understand the effects of dwell time and a tensile load level on the creep-cyclic plasticity behaviour of the PRTMCs. The cyclic creep and plastic analyses are performed for the RVE models which are subjected to each cyclic load point P1, P2, and P3 using the LMM eDSCA for three independent dwell of $1 \mathrm{hr}, 10 \mathrm{hrs}$, and $100 \mathrm{hrs}$. Figure 12 presents the hysteresis loops constructed for the most critical element of each case. Creep strain and ratchetting strain analysed are summarised in Table 4.

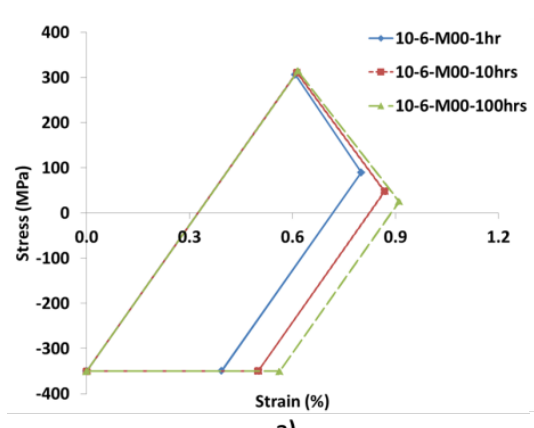

a)

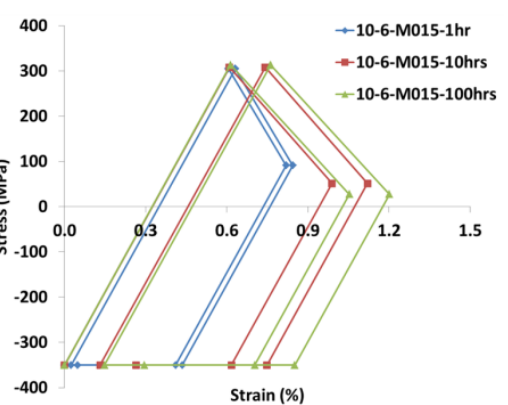

b)

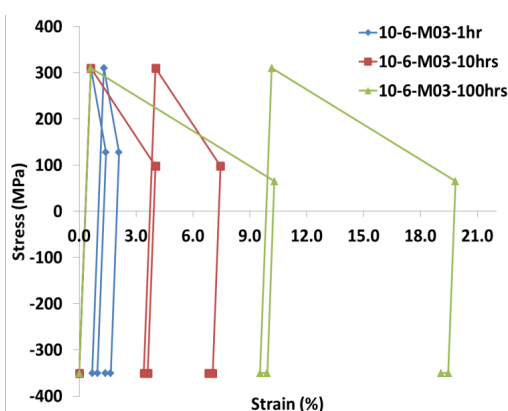

C)

Figure 12 Steady state hysteresis loops of a RVE model that has $N_{\text {part }}=6$ with $V_{f}=10 \%$ for variations of dwell 1hr, 10hrs, and 100hrs at cyclic load point; a) P1, b) P2, and c) P3.

For the non-tensile loading applied (Figure 12-a), the dwell stress relaxation affects the unloading plasticity but closed hysteresis loops appear for all the dwell times. As dwell increases, creep deformation increases, but the magnitude of the end of dwell stress decreases. Different from other thermal stress induced by non-isothermal loading over a structure, the RVE has the thermal stress caused by a difference of thermal expansion coefficients between the titanium phase and the silicon carbide in the isothermal condition. Therefore no back stress effects exist during the relaxation; the pure thermal stress will be close to zero over a long-term dwell.

For the tensile loads applied, Figure 12-b) and c), creep ratchetting responses occur at every dwell time due to the cyclically enhanced creep. In overall, creep deformations significantly increase as tensile load level increases despite the same dwell. Interesting points to be noted from are that the 
RVE models subjected to the larger tensile load level P3 have the dwell relaxation started and ended at higher stress level than the others P1 and P2. When the tensile load and the thermal load are applied to the RVE models, primary tensile stresses and secondary compressive stresses develop respectively. Critical stresses combined between the primary and secondary stresses in the RVE models are likely to occur at locations where the metal matrix encloses the particles as shown in Figure 11.
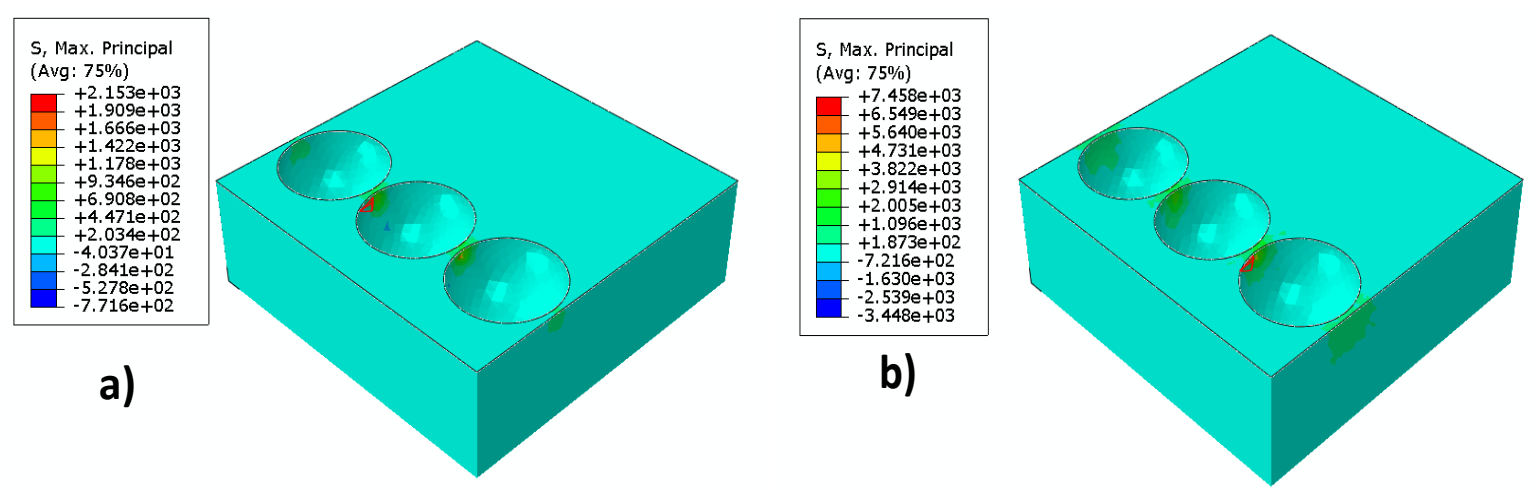

Figure 13 Maximum principal stress distributions [MPa] of a RVE model $N_{\text {part }}=6$ with $V_{f}=10 \%$ for dwell time 10hrs: a) $\sigma_{\mathrm{p}}=0$ and b) $\sigma_{\mathrm{p}}=0.15 \sigma_{\mathrm{p} 0}$

The maximum principal stress of the RVE models subjected to the three different loadings P1, P2, and P3 is investigated in order to find a dominant stress component of the von-Mises stress shown in Figure 12, the maximum principal stress component of the RVE model subjected to the three different loadings P1, P2, and P3 is investigated. Figure 13 exhibits contours of the maximum principal stress distributions for P1 and P2. The locations where the critical stress imposed have the tensile maximum principal stress component, whereas the rest of the metallic phase in the RVE model has the compressive stress component. As the tensile load increases, the start and the end dwell stress level increase, while the thermal stress being in relaxations for the same dwell. From Table 4, we can presume that creep strain may be accumulated over $1 \%$ for a dwell of an hour within a cycle, if the tensile load level exceeds P3. Therefore, creep damage should be carefully assessed when the PRTMCs is subjected to tensile loading condition along with the cyclic thermal load. 
Table 4 Creep and ratchetting strain from the creep behaviour analyses of an array of 6 particles with a fraction volume of $10 \%$.

\begin{tabular}{|c|c|c|c|}
\hline Cyclic load point & Dwell time & Creep strain (\%) & Ratchetting strain (\%) \\
\hline & $1 \mathrm{hr}$ & 0.39 & 0.00 \\
\hline \multirow[t]{3}{*}{$P 1$} & $10 \mathrm{hrs}$ & 0.50 & 0.00 \\
\hline & $100 \mathrm{hrs}$ & 0.56 & 0.00 \\
\hline & $1 \mathrm{hr}$ & 0.41 & 0.04 \\
\hline \multirow[t]{3}{*}{$P 2$} & $10 \mathrm{hrs}$ & 0.62 & 0.18 \\
\hline & $100 \mathrm{hrs}$ & 0.70 & 0.22 \\
\hline & $1 \mathrm{hr}$ & 0.93 & 0.80 \\
\hline \multirow[t]{2}{*}{ P3 } & $10 \mathrm{hrs}$ & 3.55 & 3.50 \\
\hline & $100 \mathrm{hrs}$ & 9.84 & 9.25 \\
\hline
\end{tabular}

\subsubsection{Effect of fraction volumes on the creep-cyclic plasticity response for a fixed particle arrangement}

As demonstrated in Section 4.2.1, the optimised particle distributions in the RVE models do not direct benefits to creep endurance. However, it is worth investigating what influences of the variations of the particle arrangement giving to the creep-cyclic plasticity behaviour of the PRTMCs. For the investigations, we have performed the cyclic creep and plastic analysis for RVE models that have a fixed particle distribution concerning a number of particles.

Two RVE models which have $\mathrm{N}_{\text {part }}=6$ and $\mathrm{N}_{\text {part }}=10$ with $\mathrm{V}_{\mathrm{f}}=25 \%$ each are selected as a reference particle arrangement. For each number of particles, four RVE models are created for different volume fractions of $10 \%, 14 \%, 18 \%$, and $22 \%$ with a fixed arrangement. The cyclic creep and plastic analysis is performed for the eight RVE models subjected to the cyclic load point P2 for a dwell time of $10 \mathrm{hrs}$. Figure 14 presents stress-strain hysteresis loops for the most critical element of each RVE model in the steady-state. Table 5 reports creep and ratchetting strain accumulated for each RVE model. 


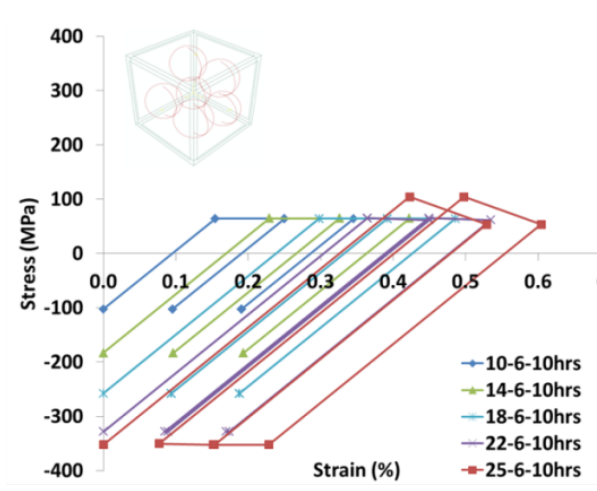

a)

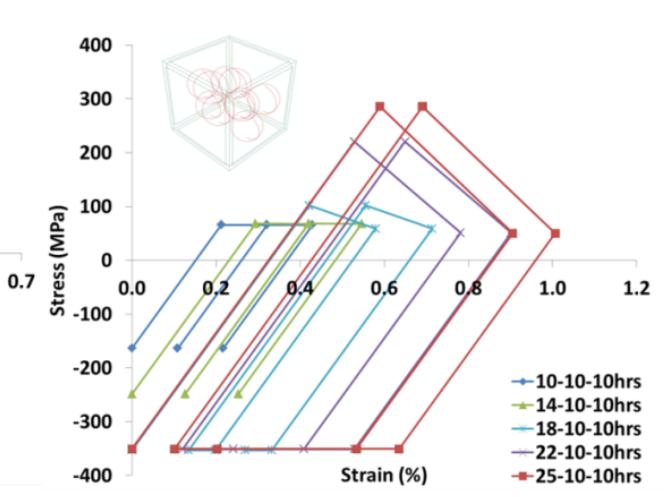

b)

Figure 14 Steady state hysteresis loops of RVE models that have a fixed particle arrangement subjected to cyclic loading $\mathrm{P} 2$ and dwell time of $10 \mathrm{hrs}$ with variations of fraction volumes: a)

$$
\mathbf{N}_{\text {part }}=6 \text { and b) } \mathbf{N}_{\text {part }}=10 \text {. }
$$

In overall, results from the both number of particles present that the cyclically enhanced creep leads to creep ratchetting response without loading plasticity. As a common trend in Figure 14, both $\mathrm{N}_{\text {part }}=6$ and $\mathrm{N}_{\text {part }}=10$ models have an increase in the stress range as volume fraction increases. An interesting point to be noted is that RVE models with volume fractions that do not induce unloading plasticity have approximately the same creep strain of $0.1 \%$.

Table 5 Creep and ratchetting strains for $N_{p a r t}=6$ and $N_{\text {part }}=10$ of $R V E$ models that have a fixed particle arrangement subjected to cyclic loading P2 and dwell time of $10 \mathrm{hrs}$ with variations of fraction volumes.

\begin{tabular}{cccc}
\hline Number of Particle & Fraction volume (\%) & Creep strain (\%) & Ratchetting strain (\%) \\
\hline \multirow{2}{*}{6} & 10 & 0.10 & 0.10 \\
14 & 0.10 & 0.10 \\
& 18 & 0.09 & 0.10 \\
22 & 0.09 & 0.09 \\
& 25 & 0.15 & 0.08 \\
\hline \multirow{2}{*}{$\mathbf{1 0}$} & 10 & 0.11 & 0.11 \\
& 14 & 0.13 & 0.13 \\
& 18 & 0.20 & 0.13 \\
& 22 & 0.41 & 0.12 \\
& 25 & 0.53 & 0.12 \\
\hline
\end{tabular}

Conversely, the creep strain increases once unloading plasticity appeared, and it keeps on increasing as the unloading plastic strain increases. By increasing the volume fraction within a fixed particle 
arrangement, a stress concentration occurs around the reinforcement due to the reduced amount of the titanium matrix. In additions, when the RVE model has the unloading plasticity accumulated, the residual stress from the unloading instance affects the start dwell stress to increase; eventually, it leads to an increase in creep strain accumulation. In the sense of the above investigations, $\mathrm{N}_{\text {part }}=10$ models may have less stress concentration around the reinforcement than $\mathrm{N}_{\text {part }}=6$ models, which results in the unloading plasticity taking place early. In the meantime, the ratchetting strains accumulated for both numbers of particles do not have any influences from the variation of volume fraction.

\section{Conclusions}

Shakedown and limit state analysis of PRTMCs subjected to thermo-mechanical loading are analysed by means of the LMM. Fifteen RVEs have been used which come from a previous study on the optimization of the elastic behaviour of particle reinforced composites by varying the particle spatial distribution. On the basis of the current investigation of the effect of particle spatial distribution, number of particles $\mathrm{N}_{\text {part }}$ and particle fraction volume $\mathrm{V}_{\mathrm{f}}$ on the two critical design limits i.e. reverse plasticity limit $\Delta \theta_{r p}$ and limit load $\sigma_{p l}$ it has been observed that:

- For a fixed particle arrangement, the reverse plasticity limit $\Delta \theta_{r p}$ decreases with $\mathrm{V}_{\mathrm{f}}$ while the limit load $\sigma_{p l}$ increases with $\mathrm{V}_{\mathrm{f}}$. Also, the array with a lower number of particles has a higher reverse plasticity and a lower limit load throughout the fraction volume range investigated.

- Instead, by comparing different arrays with the same $\mathrm{N}_{\text {part }}$ but different $\mathrm{V}_{\mathrm{f}}$ the particle spatial distribution can influence the stress at the matrix/particle interface leading to a higher value of $\Delta \theta_{r p}$ for the array with the highest $\mathrm{V}_{\mathrm{f}}$ and a higher value of $\sigma_{p l}$ for the array with the lowest $\mathrm{V}_{\mathrm{f} .}$ Likewise by comparing different arrays with the same $\mathrm{V}_{\mathrm{f}}$ but different $\mathrm{N}_{\text {part }}$ the particle spatial distribution can lead to a higher value of $\Delta \theta_{r p}$ for the array with the highest $\mathrm{N}_{\text {part }}$ and a higher value of $\sigma_{p l}$ for the array with the lowest $\mathrm{N}_{\text {part }}$

With regards to the creep-cyclic plasticity behaviours of the PRTMCs, the fifteen RVE models have been analysed by the LMM eDSCA. Key observations from the results are summarized as follows:

- All the RVE models analysed show creep ratchetting responses due to cyclically enhanced creep, despite a cyclic load applied is under the strict shakedown boundary. Despite the same number of particles, variations of volume fractions have no direct influences on creep or ratchetting endurances due to non-uniform spatial particle distribution. Therefore the 
numerical results demonstrate again that tailoring of the reinforcement arrangement affects the high temperature damage tolerance substantially. Nevertheless, the non-uniform spatial particle distribution, a cyclic loading closer to the reverse plasticity limit causes significant creep deformation. Moreover, tensile load level has significant effects on both creep strain and ratchetting strain increment as a dwell increase.

- For RVE models with a fixed particle arrangement, variations of volume fractions have effects on a magnitude of a stress range within a cycle, which enhance unloading plasticity as volume fractions increase. On the other hand, a large number of particles augment the total strain range for the same volume fraction. Therefore, an RVE model that has a smaller volume fraction exhibits outstanding creep endurance.

\section{Acknowledgement}

The authors gratefully acknowledge the supports from the National Natural Science Foundation of China (51828501), University of Strathclyde and East China University of Science and Technology during the course of this work.

\section{References}

1. Huang, L. and L. Geng, Discontinuously Reinforced Titanium Matrix Composites: Microstructure Design and Property Optimization. 2017: Springer.

2. Yang, Z., et al., In situ synthesis of hybrid and multiple-dimensioned titanium matrix composites. Journal of alloys and compounds, 2006. 419(1): p. 76-80.

3. Chen, G., A. Bezold, and C. Broeckmann, Influence of the size and boundary conditions on the predicted effective strengths of particulate reinforced metal matrix composites (PRMMCs). Composite Structures, 2018. 189: p. 330-339.

4. Kimmig, S. and J.-H. You, Cyclic plastic behavior of unidirectional SiC fibre-reinforced copper composites under uniaxial loads: An experimental and computational study. Composite Structures, 2018.

5. Tian, W., et al., Numerical evaluation on mechanical properties of short-fiber-reinforced metal matrix composites: Two-step mean-field homogenization procedure. Composite Structures, 2016. 139: p. 96-103.

6. Tian, W., et al., Numerical simulation on elastic properties of short-fiber-reinforced metal matrix composites: Effect of fiber orientation. Composite Structures, 2016. 152: p. 408-417.

7. Cai, L., et al., Research on development of in situ titanium matrix composites and in situ reaction thermodynamics of the reaction systems. Journal of University of Science and Technology Beijing, Mineral, Metallurgy, Material, 2006. 13(6): p. 551-557.

8. Ranganath, S., A review on particulate-reinforced titanium matrix composites. Journal of Materials Science, 1997. 32(1): p. 1-16.

9. Ainsworth, R., R5: Assessment procedure for the high temperature response of structures. British energy generation Ltd, 2003. 3. 
10. Chen, H., W. Chen, and J. Ure, A Direct Method on the Evaluation of Cyclic Steady State of Structures With Creep Effect. Journal of Pressure Vessel Technology, 2014. 136(6): p. 061404-061404.

11. Bree, J., Elastic-plastic behaviour of thin tubes subjected to internal pressure and intermittent high-heat fluxes with application to fast-nuclear-reactor fuel elements. The Journal of Strain Analysis for Engineering Design, 1967. 2(3): p. 226-238.

12. Barbera, D., H. Chen, and Y. Liu, Advances on creep-fatigue damage assessment in notched components. Fatigue \& Fracture of Engineering Materials \& Structures, 2017.

13. Cho, N.-K. and H. Chen. Investigation of structural creep strain recovery and its impact on structural integrity. in 24th International Conference on Structural Mechanics in Reactor Technology. 2017.

14. Cho, N.-K., et al., Enhanced fatigue damage under cyclic thermo-mechanical loading at high temperature by structural creep recovery mechanism. International Journal of Fatigue, 2018.

15. Böhm, H.J. and W. Han, Comparisons between three-dimensional and two-dimensional multiparticle unit cell models for particle reinforced metal matrix composites. Modelling and Simulation in Materials Science and Engineering, 2001. 9(2): p. 47.

16. Han, W., A. Eckschlager, and H.J. Böhm, The effects of three-dimensional multi-particle arrangements on the mechanical behavior and damage initiation of particle-reinforced MMCs. Composites science and technology, 2001. 61(11): p. 1581-1590.

17. Segurado, J., J. LLorca, and C. González, On the accuracy of mean-field approaches to simulate the plastic deformation of composites. Scripta Materialia, 2002. 46(7): p. 525-529.

18. Chen, H. and A. Ponter, The 3-D shakedown and limit analysis using the linear matching method. Int. J. Pressure Vessels Piping, 2002. 78: p. 443-451.

19. Giugliano, D., D. Barbera, and H. Chen, Effect of fiber cross section geometry on cyclic plastic behavior of continuous fiber reinforced aluminum matrix composites. European Journal of Mechanics-A/Solids, 2017. 61: p. 35-46.

20. Giugliano, D. and H. Chen, Micromechanical modeling on cyclic plastic behavior of unidirectional fiber reinforced aluminum matrix composites. European Journal of MechanicsA/Solids, 2016. 59: p. 155-164.

21. Chen, H. and A.R. Ponter, A method for the evaluation of a ratchet limit and the amplitude of plastic strain for bodies subjected to cyclic loading. European Journal of Mechanics-A/Solids, 2001. 20(4): p. 555-571.

22. Lytwyn, M., H. Chen, and M. Martin, Comparison of the Linear Matching Method to RollsRoyce's Hierarchical Finite Element Framework for ratchet limit analysis. International Journal of Pressure Vessels and Piping, 2015. 125: p. 13-22.

23. Chen, H. and A.R. Ponter, Linear Matching Method on the evaluation of plastic and creep behaviours for bodies subjected to cyclic thermal and mechanical loading. International Journal for Numerical Methods in Engineering, 2006. 68(1): p. 13-32.

24. Barbera, D., H. Chen, and Y. Liu, On Creep Fatigue interaction of components at elevated temperature. Journal of Pressure Vessel Technology, 2016. 138(4): p. 041403.

25. Beesley, R., H. Chen, and M. Hughes, A novel simulation for the design of a low cycle fatigue experimental testing programme. Computers \& Structures, 2017. 178: p. 105-118.

26. Beesley, R., H. Chen, and M. Hughes, On the modified monotonic loading concept for the calculation of the cyclic j-integral. Journal of Pressure Vessel Technology, 2015. 137(5): p. 051406.

27. Jackson, G., H. Chen, and D. Tipping, Shakedown and creep rupture assessment of a header branch pipe using the Linear Matching Method. Procedia Engineering, 2015. 130: p. 17051718.

28. Barbera, D., H. Chen, and Y. Liu, Creep-fatigue behaviour of aluminum alloy-based metal matrix composite. International Journal of Pressure Vessels and Piping, 2016. 139-140: p. 159-172. 
29. Barbera, D., H.F. Chen, and Y.H. Liu, On the Creep Fatigue Behavior of Metal Matrix Composites. Procedia Engineering, 2015. 130: p. 1121-1136.

30. Giugliano, D., et al., Creep-fatigue and cyclically enhanced creep mechanisms in aluminium based metal matrix composites. European Journal of Mechanics - A/Solids, 2019. 74: p. 6680.

31. Koiter, W.T., General theorems for elastic-plastic solids. 1960: North-Holland Amsterdam.

32. Melan, E., Theorie statisch unbestimmter Systeme aus ideal-plastischem Baustoff. 1936: Hölder-Pichler-Tempsky in Komm.

33. Gentile, L., et al., Optimisation based analysis of the effect of particle spatial distribution on the elastic behaviour of PRMMC. ECCM-6th European Conference on Computational Mechanics (Solids, Structures and Coupled Problems)-Glasgow, 2018.

34. Grace, A., Optimization Toolbox: for Use with MATLAB: User's Guide, November 1990. 1990: MathWorks Incorporated.

35. Van Rossum, G. Python Programming Language. in USENIX Annual Technical Conference. 2007.

36. Abaqus, C., Software for finite element analysis Abaqus/Complete Abaqus Environment, Version 6.12. Dassault Systémes. Vélizy-Villacoublay, 2013.

37. McKay, M.D., R.J. Beckman, and W.J. Conover, Comparison of three methods for selecting values of input variables in the analysis of output from a computer code. Technometrics, 1979. 21(2): p. 239-245.

38. Mitchell, M., An introduction to genetic algorithms. 1998: MIT press.

39. Wales, D.J. and J.P. Doye, Global optimization by basin-hopping and the lowest energy structures of Lennard-Jones clusters containing up to 110 atoms. The Journal of Physical Chemistry A, 1997. 101(28): p. 5111-5116.

40. Al Kassem, G. and D. Weichert, Micromechanical material models for polymer composites through advanced numerical simulation techniques. PAMM, 2009. 9(1): p. 413-414.

41. Giugliano, D., et al., Creep-fatigue and cyclically enhanced creep mechanisms in aluminium based metal matrix composites. European Journal of Mechanics-A/Solids, 2019. 74: p. 66-80.

42. Badea, L., et al., Creep behavior of Ti-6Al-4V from $450^{\circ} \mathrm{C}$ to $600^{\circ} \mathrm{C}$. University Polytechnica of Bucharest Scientific Bulletin, Series B, 2014. 76(1): p. 185-196.

43. Segurado, J. and J. LLorca, Computational micromechanics of composites: the effect of particle spatial distribution. Mechanics of materials, 2006. 38(8): p. 873-883.

44. Gentile, L., et al. Surrogate assisted optimization of particle reinforced metal matrix composites. in Proceedings of the Genetic and Evolutionary Computation Conference. 2018. ACM.

45. Mishnaevsky Jr, L., K. Derrien, and D. Baptiste, Effect of microstructure of particle reinforced composites on the damage evolution: probabilistic and numerical analysis. Composites Science and Technology, 2004. 64(12): p. 1805-1818.

46. Mishnaevsky, L. 3D finite element study of damage evolution in a particle composite. in ECF15, Stockolm 2004. 2004. 


\section{Appendix}

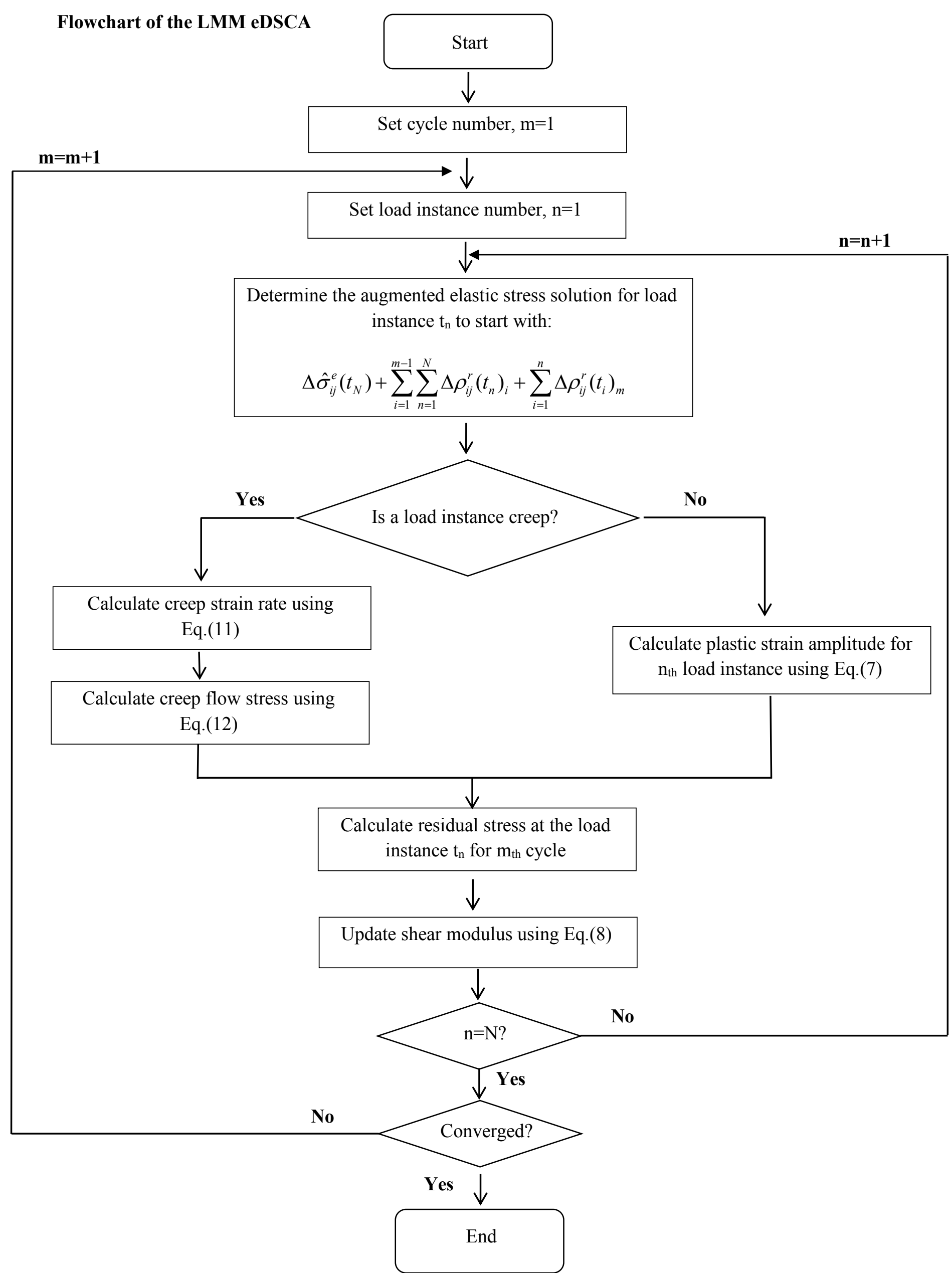

